\title{
Using digital monitoring during the COVID pandemic to streamline outpatient appointments
}

\author{
Walsh A, Matini L, Wilson J, Lyden S, Al-Hillawi L, White L, Kantschuster R, Kormilitzin A, Smith T, \\ Reilly S, Slater J, Brain AO, Palmer R, Ambrose T, Satsangi J, Travis SPL
}

\section{Background}

Demand for outpatient appointments (OPAs) for IBD often exceeds capacity, partly due to scheduled follow up of patients who are well. The TrueColours-IBD (TC-IBD) platform and Escalation of Therapy or Intervention (ETI) calculator was trialled as a tool to triage appointments during the pandemic

\section{Methods}

TC-IBD is a web-based programme of email prompts linked to validated disease-specific indices. The ETI calculator was created after logistic regression showed that patient-reported symptoms and quality of life could calculate the probability of therapy escalation or intervention during an OPA. A score $\leq 20$ equates to $\leq 10 \%$ chance of escalation

The ETI calculator was developed for UC, but it was also applied to CD during the pandemic, replacing the SCCAI score with HBI. From Mar-Oct 2020 the ETI calculator was used to extend 145 OPAs (87 UC, 58 CD) from 1034 ETI assessments. TC-IBD data was assessed 2-6 weeks before a scheduled OPA. Patients were eligible for OPA extension if $\geq 2$ symptom (SCCAI/HBI) and $1 \mathrm{QoL}$ responses (IBD Control) within 4 weeks and ETI score $\leq 20$. Patients with extended OPAs were monitored for 3 \& 6-monthly ICHOM outcomes (www.ichom.org), collected through the TC-IBD platform

\section{Results}

ICHOM outcomes available for $113 / 145$ patients at 3 mo and 125/145 patients at 6 mo. There were no emergency department visits, no hospitalisations, no surgery, colon cancer or death at either time point in patients whose appointment was extended. 1 patient with UC required prednisolone (Table 1).

Table 1: ICHOM Outcomes at 3 and 6 mo in patients with extended appointments

\begin{tabular}{|c|c|c|c|c|c|c|c|c|}
\hline & $\begin{array}{c}\text { No. patients } \\
\text { with ICHOM } \\
\text { data }\end{array}$ & $\begin{array}{l}\text { ED visits } \\
\text { (IBD) }\end{array}$ & $\begin{array}{l}\text { Hospitalisations } \\
\text { (IBD) }\end{array}$ & $\begin{array}{l}\text { Advice line contact } \\
\text { (reporting a flare) }\end{array}$ & $\begin{array}{l}\text { Prednisolone } \\
\text { (IBD) }\end{array}$ & Surgery & $\begin{array}{l}\text { Colon } \\
\text { cancer }\end{array}$ & Death \\
\hline $\begin{array}{l}3 m \\
\text { (UC) }\end{array}$ & $75 / 87$ & 0 & 0 & $10(5)$ & 1 & 0 & 0 & 0 \\
\hline $\begin{array}{l}6 \mathrm{~m} \\
\text { (UC) }\end{array}$ & $68 / 87$ & 0 & 0 & $10(7)$ & 0 & 0 & 0 & 0 \\
\hline $\begin{array}{l}3 m \\
(C D)\end{array}$ & $58 / 58$ & 0 & 0 & $5(2)$ & 0 & 0 & 0 & 0 \\
\hline $\begin{array}{l}6 \mathrm{~m} \\
(\mathrm{CD})\end{array}$ & $57 / 58$ & 0 & 0 & $6(2)$ & 0 & 0 & 0 & 0 \\
\hline
\end{tabular}

Of 12 reported flares of UC, 9 patients required medication change (vedolizumab re-induction +budesonide MMX (1), budesonide MMX (3), topical steroids (2), 5ASA up-titration +laxatives (2), laxatives (1)). Of 4 reported flares for $C D, 3$ patients required medication change (adalimumab uptitration +budesonide CIR (1), budesonide CIR (1), topical steroids (1)). Placing outcomes in context, 1536 of our patients completed ICHOM outcomes during the 6 mo period (UC 856, CD 680). 
Prednisolone was given to 58 \& 22, ED visits in 11 \& 21, hospital admission in 20 \& 21 and IBD-related operations in 4 \& 14 IBD (UC \& CD respectively)

\section{Conclusion}

Routine digital monitoring of symptoms, quality of life and PROMs can safely streamline outpatient care in IBD

Fig 1: Escalation of Therapy or Intervention Calculator

\begin{tabular}{|c|c|c|c|c|c|c|c|}
\hline SCCAI/HBI & Points & + & IBD CONTROL & Points & $=$ & TOTAL POINTS & Probability of Escalation \\
\hline 0 & 0 & & 0 & 24 & & 0 & 0.01 \\
\hline 1 & 7 & & 1 & 22 & & 13 & 0.05 \\
\hline 2 & 14 & & 2 & 21 & & 34 & 0.25 \\
\hline 3 & 21 & & 3 & 20 & & 47 & 0.50 \\
\hline 4 & 29 & & 4 & 18 & & 59 & 0.75 \\
\hline 5 & 36 & & 5 & 16 & & 80 & 0.95 \\
\hline 6 & 43 & & 6 & 15 & & 100 & 0.99 \\
\hline 7 & 50 & & 7 & 14 & & & \\
\hline 8 & 57 & & 8 & 12 & & & \\
\hline 9 & 64 & & 9 & 11 & & & \\
\hline 10 & 71 & & 10 & 9 & & & \\
\hline 11 & 79 & & 11 & 8 & & & \\
\hline 12 & 86 & & 12 & 6 & & & \\
\hline 13 & 93 & & 13 & 5 & & & \\
\hline \multirow[t]{3}{*}{$\geq 14$} & 100 & & 14 & 3 & & & \\
\hline & & & 15 & 2 & & & \\
\hline & & & 16 & 0 & & & \\
\hline
\end{tabular}

SCCAI, Simple Clinical Colitis Activity Index; HBI, Harvey Bradshaw Index; IBD-Control-8 (Bodger K et al, Gut, 2014) 